ISSN 0001-6002/2001/43/3/141-142

Acta Médica Costarricense,(C2001

Colegio de Médicos y Cirujanos

\title{
Hernia Perineal Postoperatoria: Reporte de un caso
}

Jorge Flikier-Gotlib, ${ }^{1}$ Carlos Valverde-Monge, ${ }^{1}$ Benjamín Flikier-Zelkowicz ${ }^{2}$

Resumen: La experiencia reportada mundialmente sobre hernias perineales postoperatorias es muy limitada.

Las hernias perineales, se presentan generalmente después de procedimientos en los cuales se elimina gran parte de la estructura de sostén del piso pélvico, como en una resección abdominoperineal por cáncer de recto o en exenteraciones pélvicas. Sin embargo menos del $1 \%^{1-3}$ de las resecciones abdominoperineales y menos del $10 \%{ }^{1}$ de las extracciones pélvicas producen hernias perineales que requieran reparación quirúrgica.

Presentamos a continuación nuestra experiencia con un caso, así como la técnica quirúrgica empleada. Consideramos importante presentar este caso por lo infrecuente que es la presentación de este padecimiento, así como por los malos resultados reportados mundialmente con las técnicas de reparación quirúrgica aplicadas hasta el momento, y, por el excelente resultado que obtuvimos con la técnica empleada por nosotros.

Descriptores: Hernia perineal.

Recibido: 30 de enero de 2001.

Aceptado: 5 de junio de 2001.

\section{Reporte del caso}

Se trata de un paciente masculino, de 63 años de edad, blanco, que fue sometido a una resección abdominoperineal, en diciembre de 1998, por un adenocarcinoma de recto.

Posterior a la cirugía, en agosto de 1999, presentó una hernia inguinal izquierda, por lo que se le realizó una hernioplastía con colocación de malla.

Dos años después de su resección, el paciente consulta por un cuadro de 30 días de evolución caracterizado por dolor al sentarse y la presencia de una masa en la región perineal.

Al examen físico se encuentra una masa protruyente en la región perineal, que al tacto esta cubierta por una piel muy blanda y friable, la masa se puede introducir en el canal perineal sin dificultad, y la misma sale nuevamente al pedirle al paciente que puje. Además se encuentra una hernia inguinal derecha.

\footnotetext{
1 Servicio Cirugía 2, Hospital San Juan de Dios.

$2 \quad$ Médico cirujano.
}

Correspondencia: Jorge Flikier Gotlin. Apartado 1682-1000 San José, Costa Rica. Correo electrónico: jflikier@racsa.co.cr
El paciente fue llevado a Sala de Operaciones donde bajo anestesia general, es colocado en posición ginecológica y se insertó una sonda de foley. Se realizó una incisión en la piel de la protrusión perineal. Se disecó el saco herniario, hasta el piso pélvico, resecando la porción de saco sobrante y se abrió la cavidad abdominal, y se revisó desde el periné, sin encontrar cambios patológicos. Se procedió luego a separar el peritoneo parietal de las paredes musculares del periné, se suturaron ambas paredes parietales con puntos separados en 3 capas. Luego se procedió a colocar una malla de polipropileno, la cual fue suturada con puntos de Vicryl@ a nivel del coxis, a los músculos elevadores del ano bilateralmente, y a la fascia de la región superior del periné. Por último se extirpó la piel sobrante y se realizó la sutura de piel con puntos separados. Luego se procedió a realizar la hernioplastía inguinal derecha con malla de polipropileno.

El paciente evolucionó satisfactoriamente en su postoperatorio inmediato, egresando del hospital a los 4 días postoperatorios.

Actualmente, 6 meses después de la intervención se encuentra asintomático y sin signos de recidiva. 


\section{Discusión}

Las hernias perineales son una protusión del contenido abdominal por un defecto en el piso pélvico, éstas se pueden clasificar en primarias (congénitas) y secundarias (postoperatorias). ${ }^{1}$

Las secundarias, son hernias que se producen generalmente después de una resección abdominoperineal por cáncer del recto, o prostatectomías perineales o exenteraciones pélvicas. Estas hernias se presentan por defectos en la reconstrucción del piso pélvico y sobretodo en procedimientos en los cuales se remueve gran parte del peritoneo y del soporte musculofascial del piso pélvico, ${ }^{1}$ como es el caso de las resecciones abdominoperineales. Para evitar la aparición de una hernia perineal postoperatoria, debe practicarse una sutura de los músculos del periné en aquellos pacientes en que la resección así lo permita.

La incidencia de hernias perineales sintomáticas, secundarias a una cirugía abdominoperineal, se ha calculado de un $0.62 \%^{2}$ a un $1 \%^{1-3}$ y para exenteraciones pélvicas de un $3 \% .^{2}$

Los síntomas principales de presentación incluyen la sensación de presión perineal y dolor, así como "molestias" al sentarse. En ocaciones el paciente puede presentarse con síntomas urinarios, o de obstrucción intestinal o con lesiones erosivas en la piel. ${ }^{2}$ El tiempo promedio entre el procedimiemto quirúrgico y el inicio de la presentación de síntomas se ha calculado en 5.8 años. $^{3}$

El diagnóstico es clínico, sin embargo se ha determinado que hasta en un 7\% de los pacientes a los cuales se les realiza estudios radiológicos con bario después de una cirugía abdominoperineal, presentan hernias perineales asintomáticas. ${ }^{2}$

Debido a lo poco frecuente de este padecimiento, existen pocos reportes en la literatura sobre el tratamiento del mismo, sin embargo se han descrito numerosas técnicas con resultados variables.

Entre las técnicas más utilizadas se encuentran las que utilizan la vía perineal, la vía abdominal o ambas (abdominoperineal) para el tratamiento de la hernia.

El fin del tratamiento es el mismo que para cualquier otra hernia, o sea, mobilizar el saco herniario, disecarlo y reparar el defecto.

La vía perineal permite el acceso más simple, sin embargo la zona de exposición es limitada y puede dificultar la movilización de intestino en casos de adherencias y dificultar la reparación de tejido intestinal lesionado. ${ }^{2}$

La técnica consiste en el cierre del defecto muscular, ya sea con suturas no absorbibles o la colocación de una malla sintética.
En el Massachusetts General Hospital se reportó 21 pacientes tratados entre 1963 y 1995 por hernia perineal postoperatoria y se concluyó que la mejor vía de abordaje es la perineal, y que la abdominal se debe dejar para los casos de recidiva de la misma. ${ }^{2}$

La vía abdominal permite confirmar la ausencia o recurrencia del cáncer, así como la movilización del intestino delgado con visión directa. Esta vía permite además la colocación de la malla a un nivel más alto en la pelvis, con pocas posibilidades de producir daños al suturar la misma. Un estudio de la Cleveland Clinic Foundation, de 8 pacientes con hernia perineal postoperatoria, concluyó que el mejor método para reparar este problema tan poco común, es la colocación de una malla en el área del piso pélvico por vía abdominal ya que tuvieron sólo una recurrencia. ${ }^{3}$

La vía doble (abdominoperineal) tiene las ventajas que ofrece la vía abdominal, asociado a la ventaja de poder resecar la piel sobrante del perineo.

Nosotros consideramos apropiada la vía perineal, dejando la vía abdominal para los casos de recidiva, por la alta probabilidad de formación de adherencias postoperatorias a la malla, al no quedar ésta cubierta por peritoneo.

\section{Referencias}

1. Sarr MG, Stewart JR, Cameron JC. Combined abdominoperineal approach to repair of postoperative perineal hernia. Dis Colon Rectum 1982; 25 (6): 597-9.

2. So JB, Palmer MT, Shellito PC. Postoperative perineal hernia. Dis Colon Rectum 1997; 40 (8): 954-7.

3. Beek DE, Fazio VW, Jagelman DG, Lavery IC, Mcgonagle BA. Postoperative perineal hernia. Dis Colon Rectum 1987; 30 (1): 21-4. 